\title{
El desarrollo nuclear de Argentina y el régimen de no proliferación
}

\author{
Sandra Colombo,, Cristian Guglielminotti, ${ }^{* *}$ María Nevia Vera***
}

Perfiles Latinoamericanos

Flacso México

\section{Resumen}

El desarrollo y la transferencia de tecnología nuclear han sido puestos bajo estrictos controles debido a su posible derivación hacia el uso bélico, lo que ha llevado a crear un régimen internacional de no proliferación. Una de las últimas propuestas ha sido la fundación de bancos multilaterales de uranio levemente enriquecido (ule). Este artículo plantea que el objetivo de no proliferación puede contribuir a acentuar la asimetría en la distribución del poder económico-político mundial, perjudicando el derecho al desarrollo endógeno de esa tecnología en los países emergentes. Aquí se investiga la existencia de márgenes de maniobra en el sistema internacional a partir del estudio del caso de Argentina, una de las pocas naciones emergentes que han conseguido exportar tecnología nuclear, y que ha declarado el desarrollo de la actividad nuclear independiente como política de Estado.

\begin{abstract}
The development and transfer of nuclear technology have been put under strict control due to its possible military use, leading to the creation of international non-proliferation regimes. One of the latest proposals was the creation of multilateral banks of low enriched uranium (leu). The article states that, under the goal of non-proliferation, this initiative can contribute to accentuate the asymmetry in the distribution of world economic and political power, damaging the endogenous development of this technology in emerging countries. Against this, the article investigates the existence of margins to maneuver in the international system from the case of study of Argentina, one of the few developing countries that have managed to export nuclear technology, and has declared the development of independent nuclear activity as a State policy.
\end{abstract}

Palabras clave: energía nuclear, Argentina, régimen de no proliferación, bancos de ULE, desarrollo. Keywords: Nuclear energy, Argentina, non-proliferation regime, LEu banks, development.

* Doctora en Sociología (UNEsp/Brasil). Investigadora e integrante del Consejo de Dirección del Centro de Estudios Interdisciplinarios de Problemáticas Internacionales y Locales (CEIPIL-UNCPBA). Directora de Proyectos de Investigación, Secretaría de Políticas Universitarias/Ministerio de Educación de la República Argentina.

** Magíster en Política y Gestión de la Ciencia y Tecnología por la Universidad Nacional de Buenos Aires (UBA). Investigador del Centro de Estudios Interdisciplinarios de Problemáticas Internacionales y Locales (CEIPIL-UNCPBA).

*** Maestranda en Ciencias Sociales por la Universidad Nacional del Centro de la Provincia de Buenos Aires. Investigadora del Centro de Estudios Interdisciplinarios de Problemáticas Internacionales y Locales (CEIPIL-UNCPBA). 


\section{Introducción}

- as capacidades en ciencia y tecnología se han convertido en motores de desarrollo por excelencia en las sociedades modernas al generar un acelerado proceso de dinamización de las economías de los Estados, y provocar, a su vez, una modificación de la estructura de poder en el sistema mundial (Krippendorff, 1985; Piñón, 2004). Entre las tecnologías creadas durante el siglo xx, la nuclear es un caso particular ya que además de constituirse en productora de estímulos para otros sectores estratégicos como la medicina, la industria o la generación de energía, puede tener un uso dual, es decir orientarse hacia la fabricación de armas de destrucción masiva. ${ }^{1}$

Debido a su posible derivación hacia el uso bélico, desde sus inicios el desarrollo nuclear y el traspaso de la tecnología sensible a otros Estados se enmarcaron en el régimen internacional de no proliferación, el cual tenía como sustento los lineamientos del Tratado de No Proliferación Nuclear (TNP). Este régimen se ha hecho efectivo mediante distintas instituciones como el Organismo Internacional de Energía Atómica (OIEA), el Grupo de Suministradores Nucleares (GSN) y, más recientemente, por medio de iniciativas como los Protocolos Adicionales (PA) y los bancos de uranio levemente enriquecido (ULE).

Si bien los controles y restricciones sobre la producción y transferencia del material nuclear tienen como objetivo explícito evitar que nuevos países obtengan armas nucleares, es necesario reconocer que ello también ha contribuido a que los Estados nucleares más industrializados excluyan o condicionen el acceso de los países en vía de desarrollo a tecnologías que además de promover el crecimiento de sus economías, los convertiría en eventuales competidores en los mercados de productos de alto valor agregado; esta situación ha dado lugar a un "apartheid tecnológico" (Hurtado, 2006). Frente a las restricciones impuestas por el régimen internacional a los desarrollos tecnológicos de los Estados, los países emergentes que hacen uso pacífico de la energía nuclear tienen la necesidad de encontrar márgenes de autonomía necesarios para defender sus intereses nacionales.

En este contexto, el presente artículo sostiene que el régimen de no proliferación viene implementando restricciones crecientes desde comienzos del siglo xxI, que afectan el derecho de los países al desarrollo científico y tecnológico nuclear con fines pacíficos, acentuando la asimetría en la distribución del poder global. Por otro lado, señala que a pesar de estas restricciones, algunos Estados

1 El conjunto de aparatos necesarios para enriquecer el uranio (U-235) en porcentajes aptos para fines civiles (hasta el $20 \%$ ), es el mismo que para enriquecerlo al $90 \%$, porcentaje adecuado para hacer una bomba nuclear. 
han sabido construir espacios de autonomía, mediante el establecimiento de estrategias para fortalecer sus capacidades nacionales en el dominio de tecnologías nucleares. Para demostrar estas afirmaciones, el texto se organiza en dos partes. En la primera, se presenta el régimen internacional de no proliferación y se describen los nuevos controles, en específico la creación de bancos de uranio levemente enriquecido, con el propósito de explorar si estos coartan el derecho de los países de acceder al desarrollo científico y tecnológico nuclear. En la segunda parte, se estudia el caso de Argentina, un país que desde mediados del siglo xx domina la tecnología nuclear con fines pacíficos y es uno de los once Estados soberanos en materia de energía atómica. El objetivo es identificar cuáles son los factores distintivos que posibilitaron que este país en desarrollo, que ha defendido su derecho a la independencia tecnológica, incluso del ciclo de combustible, se posicione como líder global en el área de no proliferación y reafirme su papel como proveedor confiable de material nuclear.

\section{El régimen internacional de no proliferación}

Desde la década de 1950 los países poseedores de armas nucleares han alentado la conformación del régimen internacional de no proliferación con el objetivo de reforzar el control sobre el desarrollo o la transferencia del material sensible en los Estados no nuclearmente armados, sin que ello implique el abandono del monopolio nuclear que detentan o la reducción significativa de sus arsenales.

El principal acuerdo internacional en materia de prevención de la proliferación es el Tratado de No Proliferación Nuclear (TNP), del cual son signatarios casi todos los países del mundo. ${ }^{2} \mathrm{El}$ TNP fue creado en 1968 para promover el desarme, evitar la proliferación de las armas nucleares y la tecnología armamentista, y fomentar la cooperación en el uso pacífico de la energía nuclear. El Tratado obliga a los Estados "nuclearmente armados" (aquellos que fabricaron y explotaron un artefacto nuclear antes de 1 de enero de 1967) a no proveer esos dispositivos o difundir la tecnología de su fabricación a ninguna otra nación. Mientras que los Estados "no nuclearmente armados" se comprometen a desarrollar tecnología nuclear solo para fines pacíficos y a someterse al sistema de salvaguardias del Organismo Internacional de Energía Atómica (OIEA). ${ }^{3}$

2 Con 190 Estados miembros, es el acuerdo multilateral sobre desarme nuclear con mayor alcance en el mundo. Solo tres países no firmaron el TNP, India, Pakistán e Israel, mientras que Corea del Norte se retiró del mismo en 2003.

3 Actualmente, los países que han reconocido poseer armas nucleares son los cinco integrantes del Consejo de Seguridad de las Naciones Unidas (Estados Unidos, Rusia, Reino Unido, Francia y China), además 
Vale aclarar que en los años transcurridos desde su aparición, se ha promovido enfáticamente el cumplimiento de los objetivos de no proliferación y uso pacífico de la energía nuclear aunque las exigencias han sido menores en cuanto al cumplimiento de las metas de desarme, ya que los arsenales nucleares en posesión de las potencias nucleares no solo conservan dimensiones abrumadoras sino que atraviesan una modernización, constituyéndose así en la máxima amenaza a la seguridad y a la proliferación.

Las instituciones multilaterales más importantes que ejercen estricto control sobre la producción, transferencia y comercialización del material nuclear, con el fin de garantizar el cumplimiento del TNP, son el Organismo Internacional de Energía Atómica (OIEA), y el Grupo de Suministradores Nucleares (GSN), que incluye a países exportadores de material atómico.

El OIEA es la máxima institución intergubernamental en materia nuclear. Fundado en 1957, es un órgano autónomo bajo la égida del sistema de Naciones Unidas que promueve la cooperación científica y tecnológica en el ámbito nuclear con fines pacíficos. En el artículo III de su Estatuto establece que fomentará en el mundo entero la investigación, el desarrollo y la utilización de la energía atómica con fines pacíficos aplicando salvaguardias y fijando normas de seguridad, y verificando a través de un sistema de inspección que la difusión de los conocimientos, materiales, servicios, equipo e instalaciones, no sea desviada hacia propósitos militares.

El otro organismo multilateral que ejerce control sobre la transferencia de tecnología es el Grupo de Suministradores Nucleares (GSN). Fue creado en 1975 como consecuencia de las pruebas nucleares llevadas a cabo por la India un año antes, las cuales evidenciaron que la tecnología nuclear transferida para propósitos pacíficos podía ser fácilmente derivada a usos bélicos. El GSN, integrado en la actualidad por 48 países exportadores de material nuclear, tiene como objetivo la instrumentación de directrices para regular las transferencias de tecnologías nucleares y de equipos, materiales y programas informáticos (software) de doble uso, con lo que contribuye a asegurar que tales transferencias no serán desviadas a actividades nucleares no sometidas a salvaguardias.

En la década de 1990, el descubrimiento de programas clandestinos de armas nucleares en Irak y de materiales nucleares no declarados en la República Popular Democrática de Corea demostró que un régimen de verificación eficaz debía centrarse, no solo en los materiales y actividades nucleares declarados por los propios Estados, sino también en posibles materiales y actividades no declarados. Como consecuencia, el oiEA adoptó en 1997 el Modelo de Protocolo

de India, Pakistán y Corea del Norte. Otros países han despertado sospechas de haber desarrollado armamento nuclear: Israel e Irán.

4 S. Colombo, C. Guglielminotti, M. Nevia Vera | El desarrollo nuclear de Argentina y el régimen de no proliferación Perfiles Latinoamericanos | Flacso México 
Adicional como complemento de los acuerdos de salvaguardias ya establecidos. Los protocolos adicionales, concertados sobre la base del Modelo, proveen al OIEA derechos específicos para acceder a una gran cantidad de información que está fuera del alcance de los acuerdos estándar de salvaguardias del TNP, ya que los Estados signatarios están obligados a proporcionar una información detallada sobre todos los aspectos del ciclo del combustible nuclear; permitir el uso de las tecnologías de verificación más avanzadas, y facilitar el acceso a cualquier emplazamiento nuclear $y$ a otros lugares en que haya o pueda haber materiales nucleares (Marzo, 2004; OIEA, 2008).

A comienzos del siglo XXI se produjo un incremento de la preocupación por la seguridad en la transferencia de productos nucleares debido a dos cuestiones de distinto orden. Por un lado, la nucleoelectricidad emergió como una alternativa valorable frente a las dificultades que presentaba la obtención de recursos energéticos tradicionales, evidenciándose un potencial incremento del mercado internacional de insumos nucleares. Por otro lado, los atentados del 11 de septiembre de 2001 provocaron que la comunidad internacional, con Estados Unidos a la cabeza, comenzara a preocuparse por la posible utilización de dispositivos nucleares por parte del terrorismo internacional.

El "alarmismo nuclear" (Merke \& Montal, 2009) que se produjo en los últimos ańos motivó un incremento de los controles por parte del OIEA y el GSN para evitar la proliferación, afectando la capacidad de los países de desarrollar planes nucleares. Así, para el año 2014, 101 Estados habían firmado un Protocolo Adicional debido a que se había instalado la norma de verificación estándar del TNP. Su aplicación en los países beneficiarios era considerada por el GSN como un requisito fundamental para que los países proveedores autorizaran la transferencia de instalaciones, equipos y tecnología de enriquecimiento de uranio y reprocesamiento del combustible irradiado (DerGhougassian, 2010).

Asimismo, se ha intentado limitar la obtención por parte de los países de un ciclo de combustible propio, con la idea de que los conocimientos y tecnologías sobre el enriquecimiento y el reprocesamiento de uranio son en sí mismos de naturaleza proliferante. Esto se evidencia con las llamadas iniciativas de "multilateralización del ciclo", a través de las cuales los países que cuentan con el ciclo de combustible deben proveer del recurso procesado a los que no lo tienen a través de una agencia internacional ${ }^{4}$ (Maceiras, 2009). Esta propuesta es vis-

4 Se denomina ciclo de combustible nuclear a la explotación de los yacimientos de uranio; la purificación del uranio y su conversión en material de uso nuclear, el enriquecimiento en uranio 235 del uranio natural; la fabricación de elementos combustibles; el quemado en la operación de las centrales nucleoeléctricas y de los reactores de investigación y de producción de radioisótopos; el eventual reprocesamiento de los combustibles usados; la gestión de los residuos radiactivos, y las tareas de investigación y desarrollo asociadas. 
ta por los signatarios del TNP no nucleares como una amenaza a la autonomía energética y al desarrollo industrial, y perciben que la preocupación por la no proliferación es un pretexto para limitar las oportunidades de crecimiento de países en desarrollo y establecer monopolios en un mercado en plena expansión (DerGhougassian, 2010).

Los bancos de uranio levemente enriquecido y sus posibles consecuencias para países en desarrollo

La propuesta de multilateralizar el ciclo de combustible no es nueva, ya que en 1953 el presidente de los Estados Unidos, Dwight Eisenhower, contempló la posibilidad de un banco de uranio enriquecido destinado a garantizar a todos los países el suministro del combustible nuclear (Rajaraman, 2012). La propuesta reapareció sin éxito a mediados de la década de 1970, y fue retomada en 2005, cuando aumentaron las preocupaciones por la proliferación a partir del caso iraní y por el renovado interés por la energía nuclear en un contexto de encarecimiento del petróleo (Rauf \& Vovchok, 2008). En este marco, Rusia, Estados Unidos y países de la Unión Europea han defendido la necesidad de crear bancos de uranio levemente enriquecido (ULE) para limitar la difusión internacional de la capacidad de enriquecimiento y minimizar el riesgo de la proliferación. Las reservas multilaterales tienen como finalidad garantizar el suministro de uranio enriquecido en bajo porcentaje (hasta 5\%) a aquellos países que carecen de capacidad de enriquecimiento doméstico y que aceptan el régimen de no proliferación y salvaguardias del OIEA.

Hasta el momento se han creado dos bancos de ULE, el primero fue inicialmente una propuesta rusa, y fue instituido formalmente por el OIEA y el gobierno de Rusia en marzo de 2010; está ubicado en Angarsk, en Siberia, y es propiedad de la Federación Rusa, país que lo opera y solventa. El segundo, fue aprobado por el OIEA en 2011 con emplazamiento probable en Kazajstán (OIEA, 2013). En ambos casos, el oiEa se comprometió a crear un marco que definiera la estructura de las reservas, así como supervisara el acceso de los países que necesiten del combustible. ${ }^{5}$

Los bancos de ULE han sido presentados como un "reaseguro" para aquellos países que posean o deseen generar energía nuclear para uso civil, porque establece garantías para un suministro estable y previsible (Deluchi, 2008; Rauf $\&$ Vovchok, 2008). No obstante, esta iniciativa ha provocado desconfianza res-

5 Para mayor información, véase "From Theory to Reality: The Evolution of Multilateral Assurance of Nuclear Fuel" (2011), en http://www.nti.org/analysis/articles/evolution-multilateral-assurance-nuclear-fuel/ 
pecto a cuál sería su impacto real, ya que puede afectar el principio de no discriminación en el desarrollo científico y tecnológico nuclear con fines pacíficos que fue fijado en el TNP (Art. IV) como derecho inalienable de los países. Según Sobehart (2008), la creación del banco de ULE, así como cualquier propuesta de multilateralizar el ciclo de combustible que dificulte el acceso a conocimientos, materiales, equipos e instalaciones para la investigación y la aplicación práctica de la energía nuclear para fines civiles, vulnera también el Art. III del Estatuto del OIEA que insta a fomentar y facilitar en el mundo entero el desarrollo y la implementación de tecnología, materiales y servicios nucleares con objetivos pacíficos.

A pesar de que el OIEA ha reafirmado que la elección de abastecerse a través de los bancos de Ule no implica ninguna renuncia de derechos por parte de los países receptores, varios expertos pertenecientes a diferentes países en desarrollo han hecho explícitas sus sospechas y resistencias (Horner, 2011). Por ejemplo, el especialista indio Ramamurti Rajaraman ${ }^{6}$ (2012), señala que varios países emergentes tienen dudas respecto al manejo efectivo e independiente de los bancos por parte del OIEA, temiendo que a futuro se conviertan en mecanismos que coarten sus derechos establecidos en el TNP y en el estatuto del OIEA.

En una posición semejante se ubica el vietnamita Ta Minh $\operatorname{Tuan}^{7}$ (2012), al afirmar que si bien la creación de un banco de ULE no exige en lo inmediato el abandono del derecho a enriquecer y reprocesar uranio por parte de los Estados miembros del OIEA, no existen garantías de que en un futuro no se realicen presiones por parte de las potencias, para que los países abandonen sus proyectos de avanzar en el ciclo de combustible (Ta Minh Tuan, 2012).

En la misma línea, el jordano Khaled Toukan ${ }^{8}$ (2012) expresa que la creación del banco de ule afectará el derecho de los países a enriquecer uranio o a reprocesar el combustible gastado. En su texto afirma que "sigo sin creer que, en la práctica, los bancos de combustible funcionarán como se supone fueron diseñados", ya que la finalidad última para establecer un banco no es ayudar a los Estados nucleares emergentes a que desarrollen sus sectores energéticos, sino responder a las amenazas de proliferación disminuyendo las posibilidades de que estos Estados sin armamento nuclear, desarrollen procesos estratégicos como el enriquecimiento.

Además de las cuestiones de índole política, no hay que subestimar la dimensión económica como posible incentivo a la creación de bancos de ULE,

6 Doctor en Física, copresidente del Panel Internacional de Materiales Fisionables y vicepresidente de la Academia Nacional India de Ciencias.

7 Doctor en Ciencias Políticas, profesor asociado de la Academia Diplomática de Vietnam y asesor del viceprimer ministro de ese país.

8 Doctor en Ingeniería Nuclear, presidente de la Comisión de Energía Atómica de Jordania, ex ministro de Energía y Recursos Minerales y de Educación de ese país. 
ya que las potencias nucleares se garantizarían un oligopolio en el mercado de uranio poco enriquecido, dominado en la actualidad por un reducido número de empresas: AREva (Francia), tenex (Rusia), urenco (Alemania, Países Bajos y Reino Unido), usec (Estados Unidos), Converdyn (Estados Unidos) y Cameco (Canadá) (Ruchking \& Loginov, 2006).

Teniendo en cuenta las características del sector, en cuanto al reducido número de empresas que dominan el mercado del uranio enriquecido, y su tendencia de crecimiento a futuro (OIEA, 2013), resulta entendible el argumento esgrimido por algunos expertos y países en torno a que más allá de las intenciones sostenidas por el OIEA, uno de los objetivos centrales de los bancos de uLE puede ser presionar a los países clientes para que abandonen sus intenciones de desarrollar programas propios de enriquecimiento, a cambio de un abastecimiento garantizado, con lo que se consolidaría de forma legítima el actual oligopolio mundial.

Frente a esta posibilidad, algunos países en desarrollo no están dispuestos a poner en riesgo sus derechos al enriquecimiento, establecidos en el TNP y en el estatuto del OIEA. Un argumento común por parte de los países en desarrollo que han mostrado reparos a la creación del banco de ULE, es que las potencias nucleares tan preocupadas por la proliferación, no han cumplido con el compromiso del desarme nuclear asumido con la firma del TNP, incrementando de esta forma la desigualdad en la distribución de los factores de poder en el orden internacional, al concentrar tanto el insumo básico para el desarrollo nuclear como el armamento atómico (Deluchi, 2008; Blair \& Brown, 2011). Esta es la posición mantenida por Argentina, que junto a otros Estados nucleares como Brasil, intentan defender sus derechos legítimos a la investigación científica y tecnológica, como se verá seguidamente.

\section{El desarrollo de tecnología nuclear en Argentina}

La investigación nuclear en Argentina nace en la posguerra, cuando el gobierno de Juan D. Perón crea la Comisión Nacional de Energía Atómica (CNEA) en 1950, con el objetivo de promover el estudio, desarrollo y aplicaciones en todos los aspectos vinculados a la utilización pacífica de la energía nuclear. Esta institución que se colocó bajo la órbita de las Fuerzas Armadas, fue concebida con base en los conceptos de autarquía y autonomía defendidos por el núcleo militar desarrollista que consideraba al dominio de la tecnología nuclear como un factor fundamental de prestigio y liderazgo regional e internacional (Sheinin, 2005). La protección de las Fuerzas Armadas le permitió a la industria nuclear, mantener un desarrollo permanente por más de cuarenta años, a pesar de la inestabilidad política que sacudió al país por la sucesión de gobiernos 
civiles, militares y democráticos, que implementaron alternativamente planes económicos desarrollistas y liberales sin mayor éxito y sustentabilidad.

A su vez, el desarrollo nuclear estuvo sustentando en una corriente de pensamiento conocida como pensamiento latinoamericano en ciencia, tecnología y desarrollo que, desde los años sesenta, destacó la necesidad de que los Estados generaran, a partir de políticas activas de financiamiento e infraestructura, una fuerte estructura científica y tecnológica que responda a intereses y objetivos propios de los países de la región, como forma de superar el subdesarrollo. ${ }^{9}$

En este marco favorable se fue conformando el complejo industrial nuclear, con el establecimiento de instituciones científicas y tecnológicas, como los centros atómicos Bariloche (1955), Constituyentes (1958) y Ezeiza (1967), y la creación de carreras universitarias e institutos afines para formar especialistas en el área. Se comenzó con la extracción, refinación, purificación y conversión del uranio; se inició el proceso de producción de radioisótopos para distintas aplicaciones, y se alentó la creación de empresas de alta tecnología, con el propósito principal de concurrir al desarrollo de procesos y de producción de bienes y servicios de interés para el cumplimiento del plan nuclear de la CNEA, además de ayudar a transformar el esquema productivo argentino. ${ }^{10}$

En 1968, el Poder Ejecutivo impulsó la construcción de la primera central nuclear de Argentina y de América Latina, Atucha I, destinada a abastecer de electricidad a los polos productivos del gran Buenos Aires en un contexto de gran crecimiento industrial que tornaba insuficientes las fuentes tradicionales de energía. En 1973 y 1980 se anunció la construcción de otras dos centrales nucleares, Embalse, en la provincia de Córdoba y Atucha II en la provincia de Buenos Aires. Se resolvió que estas centrales, inauguradas en 1974, 1984 y 2014, respectivamente, utilizaran uranio natural de producción nacional como combustible, debido a que las restricciones internacionales existentes dificultaban desarrollar la tecnología de enriquecimiento. ${ }^{11}$

A mediados de la década de 1980 con la consolidación del modelo neoliberal y la implementación de políticas de ajuste fiscal, los gobiernos democráticos

9 Herrera (1973) y Sábato \& Botana (1968).

10 Algunos ejemplos son: el Servicio de Asistencia Técnica a la Industria (sATI), en 1961; la Empresa Provincial de la Energía de Córdoba (EPEC), en 1967; la Empresa de Investigaciones Aplicadas, en 1976; la Empresa Nuclear Mendoza S. E. y la Empresa Nuclear Argentina de Centrales Eléctricas S. A. (ENACE, S. A.), en 1980; la Empresa Combustibles Nucleares Argentinos, S. A. (Conuar, S. A.) en 1981; Dioxiтeк, S. A; la empresa Alta Tecnología Sociedad del Estado (ALteC, S. E.), en 1985; la Fábrica de Aleaciones Especiales, S. A. (faesa) y la Empresa Córdoba Alta Tecnología, S. E. (CORATEC,) en 1986; la Empresa Neuquina de Servicios de Ingeniería, S.E. (ENSI, S.E.) para la operación de la Planta Industrial de Agua Pesada de Arroyito en 1989, Nucleoeléctrica Argentina, S. A., en 1994.

11 Es necesario hacer la salvedad que la central Atucha I fue reconvertida en 2001 para funcionar con uranio levemente enriquecido, con lo que se ahorró un $15 \%$ del costo de combustibles. 
sometieron a la industria nuclear a recortes presupuestarios que provocaron el envejecimiento de su equipamiento, la minimización de gastos operativos, y la pérdida de personal científico y técnico. A pesar de esta situación crítica, la acumulación histórica de capacidades propias, el reconocimiento público y la dimensión alcanzada, le permitieron evitar el desmantelamiento total y sortear esta etapa manteniendo un grado importante de autonomía en tecnología, recursos humanos y abastecimientos, y una proyección no solo interna sino también externa (CNEA, 2001). Probablemente hayan sido estos factores los que explican los logros alcanzados en este periodo, como la inauguración del laboratorio de celdas calientes en Ezeiza y la Fundación Escuela de Medicina Nuclear en Mendoza, en 1991; el Instituto de Tecnología en el Centro Atómico Constituyentes y la Planta Industrial de Agua Pesada de Arroyito, ${ }^{12}$ en 1993; y el Polo Tecnológico Constituyentes, en 1997.

La crisis del modelo neoliberal y la llegada a la presidencia de Argentina de Néstor Kirchner, en 2003, favorecieron el renacimiento de la industria nuclear. El rol privilegiado que adquirió la ciencia y la tecnología como soporte necesario de la nueva estrategia de desarrollo que buscaba la reindustrialización del país, junto a la necesidad de diversificar la matriz energética ante un contexto de escasez en 2004, propiciaron que la industria nuclear volviera a adquirir una importancia estratégica.

En 2006, el gobierno nacional presentó el Plan de Reactivación de la Actividad Nuclear Argentina con la finalidad de generar energía eléctrica y aplicar la energía nuclear en la salud pública y en la industria. Entre los aspectos más importantes se destacaban la decisión de finalizar Atucha II, ${ }^{13}$ la extensión de la vida útil de las centrales Embalse y Atucha I, la continuidad del proyecto CAREM, ${ }^{14}$ la construcción de dos nuevas centrales de potencia y la reactivación de la planta de enriquecimiento de uranio de Pilcaniyeu, abandonada en la década de 1990 por exigencias externas.

12 La planta se mantuvo cerrada entre 2000 y 2004, cuando reabrió sus puertas para proveer de agua pesada al reactor que Argentina vendió a Australia.

13 La construcción de la tercera central nuclear, que comenzara en 1981, fue suspendida entre 1994 y 2006 por restricciones presupuestarias. El proceso de Puesta en Marcha de la rebautizada Central Nuclear Néstor Kirchner, se inició en septiembre de 2011 y comenzó a funcionar a mediados de 2014.

14 El Proyecto Carem (Central Argentina de Elementos Modulares) le permitirá al país posicionarse a la vanguardia del mercado de centrales de baja potencia, con uranio levemente enriquecido como combustible y características relativamente sencillas en cuanto a su construcción y operación, ideales para cubrir una amplia gama de necesidades propias de los países en vías de desarrollo. El concepto CAREM nació en la década de 1980, y luego de un período de ciertas dificultades, fue declarado "de interés nacional" por el Poder Ejecutivo en 2006. En 2014 comenzó la construcción de la Central Nuclear CAREM-25, con lo que la Argentina tendrá en operación el primer reactor de potencia íntegramente diseñado y construido en el país. Recuperado de www.cnea.gov.ar 
La Argentina es uno de los pocos países que dominan el enriquecimiento de uranio junto a Estados Unidos, Reino Unido, la Federación de Rusia, Francia, China, Alemania, Pakistán, la República Popular Democrática de Corea, Japón, India y Brasil. ${ }^{15}$

Barbarán (2014) señala que, en sus orígenes, "el plan nuclear argentino no contemplaba el enriquecimiento de uranio. El país había seleccionado la línea de reactores de uranio natural con agua pesada porque consideraba que el enriquecimiento era una tecnología demasiado compleja y que presentaba demasiadas aristas complicadas en el plano internacional". Sin embargo, el endurecimiento del régimen de no proliferación a raíz de la explosión nuclear en la India (1974) y fundamentalmente, la Ley de No Proliferación Nuclear de los Estados Unidos (1978) que negaba la provisión de uranio enriquecido a países que no hubieran firmado el TNP, provocaron que los gobiernos argentinos decidieran fomentar la tecnología de enriquecimiento. Esta ley afectó particularmente a la Argentina, porque la denegación del combustible dificultó la construcción del primer reactor de investigación y producción destinado a Perú.

El método adoptado por Argentina para aumentar la concentración de uranio 235 (el isótopo capaz de producir la fisión nuclear) respecto de su porcentual en la naturaleza, fue el de difusión gaseosa ya que el método alternativo de centrifugación requería de un desarrollo tecnológico autónomo inaccesible para el país en esos años. Esta tecnología de enriquecimiento de uranio se alcanzó en 1983, gracias al trabajo conjunto de la CNEA y la empresa estatal INVAP, s. A., en la planta de enriquecimiento ubicada en el complejo Pilcaniyeu, en la provincia de Río Negro.

Simultáneamente, en 1980 el gobierno nacional declaró de interés nacional la construcción de una planta para producir agua pesada, el elemento indispensable para moderar y refrigerar el funcionamiento de los reactores nucleares. La Planta Industrial de Agua Pesada (PIAP) fue construida en la localidad de Arroyito en la provincia de Neuquén y produjo por primera vez agua pesada en 1994, ubicando a la Argentina en el reducido grupo de países con conocimientos y capacidad para producir este elemento en escala industrial y con calidad suficiente para su empleo en cualquiera de sus aplicaciones conocidas.

A pesar de estos éxitos tecnológicos, la PIAP estuvo paralizada desde el año 2000 y el complejo Pilcaniyeu fue prácticamente desmantelado en los años noventa, producto de las dificultades económicas derivadas de la implementación

15 Irán suspende el enriquecimiento a más del 20\% a comienzos de 2014. Información extraída de World Nuclear Fuel Facilities. Recuperado de http://www.wise-uranium.org/efac.html\#NFB 
de políticas afines con el Consenso de Washington y de una política exterior de "alineamiento" con las grandes potencias.

Esta situación se mantuvo hasta que los gobiernos de Néstor Kirchner y Cristina Fernández impulsaron nuevamente el desarrollo nuclear nacional. Así, en 2006, se decidió en el marco del Plan Nuclear Argentino, la reapertura del Complejo Tecnológico de Pilcaniyeu, que tras reacondicionar y actualizar sus instalaciones, fue reinaugurado en 2010.

La CNEA ha expresado que la producción nacional (exploración y enriquecimiento) permitirá dejar de importar uranio de la ex república soviética de Kazajstán, entre otros proveedores, lo que redundará en una disminución importante de los costos y en la creación de fuentes de trabajo para cientos de personas (CNEA, 2007). Por su parte, el gobierno nacional asumió que el enriquecimiento de uranio es fundamental para que la Argentina logre controlar el ciclo completo del combustible y para que alcance una independencia tecnológica que permita generar energía nuclear con usos pacíficos, ubicándola en el selecto grupo de once países que controlan este tipo de tecnología.

Otro hito fundamental se produjo en 2004, cuando comenzó a producir nuevamente la PIAP asegurando la disponibilidad de un insumo esencial para la continuidad operativa de las centrales nucleares Atucha I y Embalse. Asimismo, este relanzamiento implicó la devolución de la carga inicial de la Central Nuclear Embalse a Canadá; la exportación a Corea, Estados Unidos y Europa; la disponibilidad del insumo para el reactor de investigación exportado a Australia; la producción de la carga inicial para la Central Nuclear Atucha II y la posibilidad de producir en el futuro la carga necesaria para la cuarta central nuclear que tendrá tecnología CANDU. ${ }^{16}$

\section{Argentina como proveedor internacional de tecnología nuclear}

Para convertirse en uno de los pocos países en desarrollo que exportan tecnología nuclear, Argentina debió superar las sospechas de la comunidad internacional y demostrar que es un Estado confiable. Durante el periodo de Guerra Fría el país había sido considerado un Estado umbral, ${ }^{17}$ debido a que si bien se había adherido al OIEA en 1957 se había negado a ratificar los Tratado de Tlatelolco y el TNP. Esta situación se modificó radicalmente en la década de

16 CANDU hace referencia a las centrales que funcionan con uranio natural o con muy bajos niveles de enriquecimiento, y agua pesada como refrigerante.

17 Un Estado umbral es aquel que tiene la capacidad tecnológica de producir un explosivo nuclear en un tiempo relativamente corto si se lo propusiera (Fernández, 2010). 
1990, cuando se produjo una total adscripción de Argentina al régimen de no proliferación. Con la caída del bloque soviético y la aparición de un orden mundial unipolar, el gobierno nacional ejecutó una política exterior de alineamiento con Estados Unidos y de acuerdo con los regímenes del primer mundo como medio para alcanzar la inserción en la economía globalizada. Como consecuencia, en el ámbito nuclear, el objetivo fue dotar de absoluta transparencia a las actividades que se realizaban en Argentina y acceder a los mercados internacionales.

En el ámbito interno esto se tradujo en el abandono del desarrollo de la tecnología de enriquecimiento de uranio, y en la adaptación de la regulación de las actividades nucleares a las directrices y recomendaciones del OIEA. En este sentido, en 1997 mediante la "Ley Nacional de la Actividad Nuclear" (No 24.804) se establece que el Estado nacional fijará la política nuclear y desarrollará funciones de investigación y desarrollo a través de la CNEA y que ejercerá las funciones de regulación y fiscalización mediante la "Autoridad Regulatoria Nuclear", recientemente creada (Maceiras, 2010).

En el ámbito internacional esto derivó en la adhesión al régimen de no proliferación, desarme y control de transferencias de tecnologías sensibles, como la Declaración de Mendoza sobre Armas Químicas y Biológicas (1991), el Grupo Australia sobre Control de Trasferencias de Precursores Químicos y Biológicos (1992), el Régimen de Control de Tecnología Misilística (MTCR) (1992), el Grupo de Suministradores Nucleares (GSN) (1994), el Tratado de Tlatelolco (1994) y el Tratado de No Proliferación (1995), la Convención sobre Asistencia en Caso de Accidentes Nucleares o Peligros Radiológicos (1994), la Convención sobre la Prohibición de las Armas Químicas (CAQ) (1995), los regímenes del Comité Zangger (1995) y Acuerdos Wassenaar (1996), el Tratado de Prohibición Completa de los Ensayos Nucleares (ствт) (1996), el Kedo (1996), la Convención Conjunta Sobre Seguridad del Combustible Gastado y Sobre Seguridad en la Gestión de Desechos Radiactivos (1997), la firma del Acuerdo Regional para la Cooperación y Promoción de la Ciencia y Tecnología Nucleares en América Latina y el Caribe (ARCAL) (1998), y la sanción de la Convención sobre la Protección Física de los Materiales Nucleares (1998), entre otros.

Ya en el nuevo milenio, el orea firmó con el gobierno nacional el Acuerdo de Largo Plazo para que la Argentina actúe como Centro de Capacitación Regional en América Latina y el Caribe para la Seguridad Nuclear, Radiológica, del Transporte y de los Desechos (Mreyc, 2008). Asimismo, Argentina es uno de los 53 Estados que participan en las Cumbres de Seguridad Nuclear, que a instancias del presidente de Estados Unidos, Barak Obama, se han reunido en Washington (2010), Seúl (2012) y La Haya (2014). 
En la política de inserción internacional de Argentina, la vinculación con Brasil fue un capítulo especial, ya que desde mediados de los ańos ochenta, ambos países firmaron acuerdos de cooperación y crearon organismos conjuntos, para aliviar las presiones internacionales que procuraban "ahogar" los programas nucleares aduciendo una posible carrera bélica entre ambos Estados (Orstein, 1999). En 1990 crearon la Agencia Brasileño-Argentina de Contabilidad y Control de materiales nucleares (ABACC), con el objetivo de permitir inspecciones in situ de las centrales nucleares de ambos países y mantener un inventario del material nuclear en cada país, y en 1991 se firmó el Acuerdo Cuatripartito entre Argentina, Brasil, la ABACC y la OIEA para la aplicación de salvaguardias totales en todas sus instalaciones nucleares.

Este entendimiento tuvo una nueva manifestación en 2008 cuando se creó la Comisión Binacional de Energía Nuclear (COBEN), para discutir una estrategia de cooperación futura e identificar proyectos concretos de cooperación como la construcción de dos reactores gemelos en Argentina y Brasil, el RA-10 y el RMB (Reactor Multipropósito Brasilero), respectivamente. Según Waisman (2010: p. 49), Argentina y Brasil decidieron cooperar en el área nuclear para alcanzar mayores "grados de autonomía en el desarrollo de sus programas nucleares y conseguir ciertos avances en sus objetivos de desarrollo tecnológico, que de manera unilateral les implicaría un esfuerzo mucho mayor".

La Argentina también potenció la firma de convenios de uso pacífico de la energía nuclear con Argelia, China, Guatemala, Cuba, Turquía, Egipto, Indonesia, Rusia, Francia, Canadá, Estados Unidos, Comunidad Europea de Energía Atómica (euratom), Marruecos, Grecia, Tailandia, Bulgaria, Australia, Vietnam, Libia, Sudáfrica, India, México y Uruguay.

El histórico desarrollo de esta tecnología alejado de las armas nucleares y la proliferación permitió que Argentina se instalara como un proveedor confiable de tecnología para un número creciente de mercados. Se construyeron reactores de investigación y producción para Perú (1977/1988), Argelia (1988), Egipto (1998), Australia (2005), se firmó en 2009 la construcción de un reactor con Canadá, y se fabricaron elementos combustibles para Holanda, Australia y Polonia. Asimismo, en los últimos años, la cancillería ha trabajado para que Argentina se afiance como productor y exportador de tecnología e insumos sensibles, abriendo nuevos mercados en países como Vietnam, China, Tailandia, Túnez, Argelia, Turquía, Arabia o Emiratos Árabes.

En este punto es necesario señalar que durante los gobiernos de Néstor Kirchner y Cristina Fernández, a pesar de la importancia estratégica que adquirió el desarrollo nuclear, la adscripción a la normativa internacional sobre seguridad y no proliferación, no fue absoluta como sucedió en los años noventa. 
Por ejemplo, Argentina junto a Brasil, fueron los únicos países del GSN que se negaron a firmar un Protocolo Adicional, a riesgo de ver restringidas las posibilidades de participación en el mercado internacional, señalando que antes de imponer nuevas limitaciones a la transferencia de tecnología nuclear, se debía establecer un calendario de desarme que garantizara la reducción significativa del armamento atómico (Hibbs, 2010). Asimismo, ambos países argumentaron que un Protocolo Adicional era peligroso porque podía transformarse en una herramienta de espionaje industrial de los Estados nucleares avanzados; e innecesario, ya que ambos países habían dado muestras suficientes de transparencia y vocación pacifista adscribiendo al Tratado de Tlatelolco, creando la ABACC y firmando el Acuerdo Cuatripartito.

La posición común mantenida por Argentina y Brasil permitió una importante victoria en 2011, cuando finalmente el GSN reconoció al Acuerdo Cuatripartito como "criterio alternativo al Protocolo Adicional del oiEA", lo que significó un reconocimiento de ambos países como jugadores legítimos en el entramado nuclear internacional.

En relación a las tendencias normativas para evitar la proliferación, Argentina también ha expresado disidencias respecto a la conformación de bancos de ULE, ya que estas iniciativas de multilateralización del combustible podrían afectar el libre derecho de los países a desarrollar los usos pacíficos de la energía nuclear en todas las etapas.

\section{Argentina y los bancos de ULE: ¿Una amenaza al desarrollo tecnológico nacional?}

En cuanto a este tema, la posición de Argentina en las instituciones multilaterales se centra en la exhortación a la eliminación de las armas nucleares bajo los principios de transparencia, irreversibilidad y verificación de las medidas de desarme nuclear, más que a la creación de nuevas restricciones al desarrollo tecnológico. En este sentido, promueve la entrada en vigor del Tratado de Prohibición Completa de los Ensayos Nucleares (СтвT, por sus siglas en inglés), y desde la Comunidad de Estados de América Latina y el Caribe (CELAC), apoya la creación de un instrumento internacional jurídicamente vinculante que prohíba las armas nucleares. ${ }^{18}$

La Argentina ha manifestado que cree fervientemente en el libre derecho de los países a desarrollar los usos pacíficos de la energía nuclear, en todas las etapas, incluido el ciclo de combustible, en cumplimiento de los compromisos internacionales asumidos. Ha señalado también que no apoyará ninguna iniciativa que pretenda limitar la soberanía estatal y la propiedad y control independien-

18 Declaración de la CELAC sobre Desarme Nuclear, 20/8/2013.

S. Colombo, C. Guglielminotti, M. Nevia Vera | El desarrollo nuclear de Argentina y el régimen de no proliferación Perfiles Latinoamericanos | FLACso México 
te de un sector tecnológico clave, como es el del ciclo de combustible nuclear, restringiendo los beneficios derivados a unos pocos países, y alterando el marco político, tecnológico y legal establecido para el uso pacífico de la energía nuclear.

En la III Cumbre de Seguridad Nuclear, realizada en La Haya en marzo de 2014, Argentina, reivindicó el derecho inalienable al desarrollo de la tecnología nuclear con fines pacíficos, y ratificó su compromiso con los más altos estándares en materia de seguridad física y tecnológica, como lo demuestra la reconversión de los reactores de investigación para que funcionen con uranio de bajo enriquecimiento.

Por lo tanto, en distintos foros multilaterales, el país ha sostenido que para controlar la proliferación nuclear no hay que restringir el acceso a la tecnología a los países no proliferantes sino establecer un régimen de verificación lo más extendido y profundo posible que asegure el uso correcto de esta tecnología. De esta manera, la Argentina busca evitar — como también lo hace Brasil— que esta iniciativa cercene el acceso al conocimiento y al desarrollo pacífico de esta tecnología sensitiva profundizando las desigualdades ya existentes entre los países del sistema internacional. El peligro de ahondar la brecha se incrementa además con la posibilidad de que los poseedores de armas nucleares no cumplan con los acuerdos de desarme (Sobehart, 2008).

De acuerdo a estos postulados, cuando se define la instalación de un banco de ULE en el seno del OIEA, Argentina votó en contra de la implementación de una reserva de uranio en la zona de Angarsk, Siberia en 2009, y se abstuvo en la votación para establecer un banco internacional de uranio levemente enriquecido bajo control del oIEA en $2010 .^{19}$

La abstención (y no el rechazo) se debió a que esta propuesta, especificaba por primera vez, que los Estados no debían abandonar sus actividades nucleares pacíficas. Tal reaseguro resulta conveniente para Argentina en el presente contexto de reactivación de la actividad nuclear, puesto que como se ha visto a lo largo de este artículo, no está en la agenda del país renunciar al desarrollo de tecnología para completar el ciclo de combustible nuclear, que le garantiza mayor independencia frente a los países que dominan este nicho comercial a través de pocas empresas.

Sin embargo, más allá de esta flexibilización en la posición de Argentina, la abstención expresada pone de manifiesto que continúan existiendo dudas y sospechas sobre la posible implementación de medidas restrictivas en el futuro.

19 De 35 integrantes de la Junta de Gobernadores, el órgano ejecutivo del oIEA, 28 votos fueron a favor, seis fueron abstenciones y hubo una única ausencia: Pakistán. Entre las abstenciones se encontraron países latinoamericanos como Ecuador, Argentina, Brasil y Venezuela, mientras que Perú y Chile votaron a favor de la propuesta. 
En este marco, a mediados de 2014, el complejo Pilcaniyeu —con licencia del OIEA - comenzó a enriquecer uranio con intención de hacerlo a escala industrial al año siguiente. Si bien el método con el que se trabaja es la difusión gaseosa, tanto en el Centro Atómico Bariloche como en el Instituto para la Investigación Científica y Técnica para la Defensa (CITEDEF), se está experimentando con el método láser, lo último en tecnología de enriquecimiento.

Como es posible observar, Argentina está intentando con estas acciones de política exterior, garantizar el desarrollo de la tecnología de enriquecimiento endógena. La pregunta clave es si podrá desarrollar autónomamente tecnologías consideradas estratégicas y evitar las presiones de los poderosos del sistema internacional, que detrás de argumentos de no proliferación, defienden sus posiciones hegemónicas en el acceso y uso de este recurso estratégico.

\section{Conclusión}

Como se observó en los párrafos precedentes, Argentina ha sostenido un importante desarrollo de la actividad nuclear con fines exclusivamente pacíficos, posicionándose como líder global en el área de no proliferación y en un actor importante en el mercado internacional de tecnología y materiales nucleares.

Este desarrollo ha tenido características singulares. En principio, el sector recibió el apoyo y la protección de actores sociales clave del país con capacidad de influir sobre las políticas estatales, lo que le posibilitó una continuidad de más de sesenta años a pesar de los cataclismos económicos y políticos de la historia nacional. En segundo lugar, se sustentó en una sólida institucionalidad, ya que la CNEA demostró capacidad y prestigio para sostener las políticas de desarrollo nuclear y afrontar las presiones externas. Por otra parte, el efecto derrame sobre otras áreas de la industria nacional junto al prestigio internacional que el país adquirió gracias a esta actividad, provocaron que la sociedad argentina valore al sector atómico como un componente estratégico para el desarrollo nacional. Por último, existió una búsqueda casi permanente de autonomía. Esta aspiración del desarrollo nuclear independiente obligó a definir estrategias en el ámbito internacional para superar las medidas restrictivas de las potencias, enmarcadas en los regímenes internacionales de seguridad y no proliferación.

En relación a esto último, hubo dos aspectos claves. Por un lado, la adscripción al régimen de no proliferación, que si bien se realizó en los años noventa con una lógica de alineamiento con Estados Unidos que fue abandonada posteriormente, permitió que Argentina fuera percibida como un Estado confiable por la comunidad internacional. Por otro lado, fue de vital importancia la in- 
tegración y cooperación con Brasil, ya que permitió avanzar en el desarrollo de sus programas nucleares conservando espacios de autonomía al negociar desde una posición de mayor fortaleza frente a la comunidad internacional.

$\mathrm{Al}$ respecto, se observó que en los últimos años en el marco del régimen de no proliferación, se adoptaron medidas para controlar o limitar la producción y traspaso de tecnologías sensibles a través de normas de seguridad como los protocolos adicionales al TNP o las restricciones al desarrollo del ciclo del combustible nuclear. Esta última propuesta que incluye la creación de reservas o bancos de uranio poco enriquecido bajo el control de los Estados productores del combustible y el OIEA, ha sido considerada por varios países en desarrollo como una nueva forma solapada de perpetuar la estructura de poder mundial. Según esta concepción, la creación de bancos de ule puede generar una nueva brecha internacional donde naciones proveedoras terminarán suministrando el combustible a naciones demandantes, evitando o al menos obstaculizando, que estos países desarrollen su propia tecnología y restringiendo por lo tanto a unos pocos, los potenciales beneficios económicos derivados de estas actividades. Esta iniciativa pareciera demostrar que las potencias no están dispuestas a renunciar al control sobre un mercado de tecnología que tiene mucho de prometedor, ni a terminar con la posesión de las armas nucleares como factor disuasivo.

La Argentina de los gobiernos de Néstor Kirchner y Cristina Fernández no respaldó estas normas de seguridad orientadas a evitar la proliferación, y ha reiterado en distintos ámbitos internacionales que el desarrollo científico y tecnológico nuclear es un derecho inalienable de los Estados. Más aún, estos gobiernos han sostenido que el dominio de las tecnologías de enriquecimiento de uranio es un asunto estratégico de la nación para continuar con los desarrollos en todo el campo nuclear sin restricciones, buscando soluciones a los desafíos energéticos argentinos del siglo xxI. En función de este posicionamiento, y en un contexto internacional donde se anunciaban restricciones para controlar el ciclo de combustible nuclear, Argentina reactivó el complejo Pilcaniyeu para enriquecer uranio, se negó junto a Brasil a firmar el Protocolo Adicional al TNP y no apoyó la conformación de bancos de ULE.

Como contraparte, Argentina ha afirmado que para alcanzar el objetivo de la no proliferación, el esfuerzo no debe estar direccionado a limitar los desarrollos tecnológicos autónomos de los Estados que hacen uso pacífico de la energía nuclear, sino a medidas concretas y adicionales de desarme nuclear, conforme a los compromisos asumidos por los países poseedores de armas atómicas en el Tratado de No Proliferación. En consecuencia, Argentina ha mantenido su posición respecto a la multilateralización del ciclo de combustible, actuando de forma coordinada con Brasil, haciendo uso de las herramientas de representa- 
ción correspondientes para dejar en claro su postura en los foros internacionales, y apelando al derecho inalienable de todos los Estados a desarrollar tecnología nuclear para fines pacíficos.

Como última reflexión, el caso de Argentina muestra que aun en el marco de un régimen internacional tan estricto como el existente en el campo nuclear que condiciona fuertemente las políticas de desarrollo, los países —aun los emergentes - pueden encontrar márgenes de autonomía necesarios para defender sus intereses nacionales, tornando relevante la acción de cada gobierno en la búsqueda de caminos propios.

\section{Bibliografía}

Barbarán, G. (2014, mayo). Enriquecimiento. Por qué hay que hacerlo. Revista U238. Teconología Nuclear para el Desarrollo. Recuperado de http://u-238.com.ar/enriquecimiento-porque-hay-que-hacerlo/

Blair, B. \& Brown, M. (2011, junio). World Spending on Nuclear Weapons Surpasses \$1 Trillion per Decade. Global Zero.

Comisión Nacional de Energía Atómica (CNEA). (2007). La Argentina volverá a producir uranio tras diez años sin actividad. Recuerado de http://www.cnea.gov.ar/noticia.php?id_ noticia $=285$

Deluchi, F. (2008, abril). Pertenecer o no pertenecer, esa es la cuestión. Centro Latinoamericano de Investigaciones Científicas y Técnicas (CLICET). Recuperado de http://www.cienciayenergia.com/Contenido/pdf/080430_i_fd_tn.pdf

Derghougassian, K. (2010). No proliferación y Estrategia Nuclear. En Más allá del TNP: las oportunidades y los riesgos del futuro inmediato de la tecnología nuclear. Cuadernos de Actualidad en Defensa y Estrategia. Ministerio de Defensa de la Nación. Argentina. Recuperado de http://www.mindef.gov.ar/publicaciones/todas/cuadernos6.html

Fernández, J. (2010). Importación de tecnologías capital-intensivas en contextos periféricos: el caso de Atucha I (1964-1974). Revista CTS, 6(16).

Herrera, A. (1973, septiembre-diciembre). La creación de tecnología como expresión cultural. Revista Nueva Sociedad, (8-9).

Hibbs, M. (2010). El Grupo de Proveedores Nucleares y el protocolo Adicional del OIEA. Recuperado de http://npsglobal.org/esp/component/content/article/151-analisis/1013-grupoproveedores-nucleares-protocolo-adicional-oiea.html 
Horner, D. (2011). AIEA Board Approves Fuel Bank Plan. Arms Control Association. Recuperado de https://www.armscontrol.org/act/2011_01-02/Fuel\%20Bank

Hurtado de Mendoza, D. (2006). Ante el riesgo de un apartheid tecnológico. Recuperado de http://www.lanacion.com.ar/785793-ante-el-riesgo-de-un-apartheid-tecnologico

Krippendorff, E. (1985). Las relaciones internacionales como ciencia. México: Fondo de Cultura Económica.

Marzo, M. (2004). Protocolo adicional: lógica e impacto. ABACC,

Merke, F. \& Montal, F. (2009). El programa nuclear de Brasil ante los nuevos incentivos de la sociedad internacional. En Más allá del TNP: las oportunidades y los riesgos del futuro inmediato de la tecnología nuclear. Cuadernos de Actualidad en Defensa y Estrategia. Ministerio de Defensa de la Nación. Argentina. Recuperado de http://www.mindef.gov.ar/publicaciones/ todas/cuadernos6.html

Ministerio de Relaciones Exteriores y Culto. (2008). Acuerdo de Largo Plazo para Prestar Apoyo a la Autoridad Regulatoria Nuclear de la Argentina como Centro de Capacitación Regional en América Latina y el Caribe para la Seguridad Nuclear, Radiológica, del Transporte y de los Desechos. Recuperado de http://tratados.mrecic.gov.ar/tratado_ficha.php?id=9321

OIEA. (2013). Examen de la tecnología nuclear 2013. Conferencia General. Recuperado de http://www.iaea.org/About/Policy/C/GC57/GC57InfDocuments/Spanish/gc57inf-2_sp.pdf

OIEA. (2011, 9 de junio). Circular Informativa. Recuperado de http://www.iaea.org/Publications /Documents/Infcircs/2011/Spanish/infcirc818_sp.pdf

OIEA. (2008). Acuerdos de salvaguardias y protocolos adicionales. Recuperado de http://www.iaea .org/Publications/Booklets/Spanish/safeguards0408_sp.pdf

Piñon, F. (2004). Ciencia y tecnología en América Latina: una posibilidad para el desarrollo. En Temas de Iberoamérica. Globalización, ciencia y tecnología, v. 2, OEI.

Rajaraman, R. (2012). A pesar de los reparos, los bancos de combustible son prometedores. Bulletin of the Atomic Scientists. Recuperado de http://thebulletin.org/ node/6274

Rauf, T. \& Vovchok, Z. (2008, junio). Combustible para pensar. Boletín del OIEA, 49(2). Recuperado de http://dialnet.unirioja.es/servlet/articulo?codigo=2746949

Ruchkin, S. \& Loginov, V. (2006, septiembre). Garantizar el ciclo del combustible nuclear: ¿Cuál es la próxima etapa? Boletín del OIEA, 48(1), 24-26.

20 - S. Colombo, C. Guglielminotti, M. Nevia Vera | El desarrollo nuclear de Argentina y el régimen de no proliferación Perfiles Latinoamericanos | FLacso México 
Sábato, J. \& Botana, N. (1968, noviembre). La Ciencia y la Tecnología en el desarrollo futuro de América Latina. Revista de Integración, (3).

Sobehart, L. (2008). Perspectiva argentina sobre el Marco Multilateral del Ciclo del Combustible Nuclear del oiEa y la no proliferación nuclear. Comisión Nacional de Energía Atómica, 8(29-30), 32-34.

Toukan, K. (2012). A pesar de las garantías, persisten las dudas. Bulletin of the Atomic Scientists. Recuperado de http://thebulletin.org/\%C2\%BFes-un-banco-de-combustible-nuclear-unabuena-inversi $\% \mathrm{C} 3 \% \mathrm{~B} 3 \mathrm{n} /$ pesar-de-las-garant $\% \mathrm{C} 3 \% \mathrm{AD} a s-$ persisten-las-dudas

Tuan, T. M. (2012). Decisiones nacionales, intereses nacionales. Bulletin of the Atomic Scientists. Recuperado de http://thebulletin.org/node/6275

Waisman, V. (2010). Argentina y Brasil: Percepciones y posturas actuales frente al régimen de no proliferación nuclear. Revista Política Hoje, 19(2).

Recibido el 28 de septiembre de 2014. Aceptado el 27 de octubre de 2015. 